Pbilosophia Philosophia Scientiæ

Scientie Travaux d'histoire et de philosophie des sciences

CS 6 | 2006

Constructivism: Mathematics, Logic, Philosophy and Linguistics

\title{
Type Theory and Universal Grammar
}

\section{Aarne Ranta}

\section{(2) OpenEdition \\ Journals}

Electronic version

URL: http://journals.openedition.org/philosophiascientiae/415

DOI: 10.4000/philosophiascientiae.415

ISSN: $1775-4283$

Publisher

Éditions Kimé

\section{Printed version}

Date of publication: 1 September 2006

Number of pages: 115-131

ISBN: 2-84174-399-3

ISSN: 1281-2463

Electronic reference

Aarne Ranta, "Type Theory and Universal Grammar", Philosophia Scientiæ [Online], CS 6 | 2006, Online since 01 September 2009, connection on 15 January 2021. URL: http://journals.openedition.org/ philosophiascientiae/415; DOI: https://doi.org/10.4000/philosophiascientiae.415 


\title{
Type Theory and Universal Grammar
}

\author{
Aarne Ranta ${ }^{1}$ \\ Chalmers University of Technology and Göteborg University
}

\begin{abstract}
The paper takes a look at the history of the idea of universal grammar and compares it with multilingual grammars, as formalized in the Grammatical Framework, GF. The constructivist idea of formalizing mathematics piece by piece, in a weak logical framework, rather than trying to reduce everything to one single strong theory, is the model that guides the development of grammars in GF.
\end{abstract}

\footnotetext{
${ }^{1}$ This paper is based on a talk given at Nordiskt Filosofimöte in Helsinki, October 2002, and the Type Theory Workshop in Leiden, February 2004. The author is grateful to Per Martin-Löf and Bengt Nordström for comments. The work was supported from grant 2002-4879, Records, Types and Computational Dialogue Semantics, from Vetenskapsrådet.
} 


\section{Universal Grammar}

\subsection{Medieval Ideas}

Universal grammar is an idea often attributed to medieval philosophy. There are two famous quotes, appearing in [Gilson 1922], and later requoted in [Lyons 1968]:

Grammar is substantially the same in all languages, even though it may undergo in them accidental variations.

(Roger Bacon, 13th century)

He who knows grammar in one language, also knows it in another as far as the essentials are concerned. The fact that he cannot, however, speak another language, or understand those who speak it, arises from the difference of words and their formations, which is accidental to grammar.

(Anonymous, 12th century)

Universal grammar was severely criticized in the Renaissance time by scholars such as Alexander Hegius and Erasmus. For many contemporary linguists, it is a notion that only an "armchair linguist" can maintain.

In the anonymous 12th century quote, languages are said to differ only as for "words and their formations". Even a quick translation experiment would show a sense in which this cannot be true: an English four-word utterance is translated into a Finnish one-word utterance,

$$
\text { also in my hoúse }=\text { talossanikin. }
$$

Moreover, the stress on the word house is important, since with another stress, the Finnish translation has two words:

also in mý house $=$ minunkin talossani.

Nonetheless, we will see later that the idea that languages only differ as for "words and their formations" does make some sense, after all.

\subsection{Universal Language in Descartes and Leibniz}

In a letter to Mersenne in 1629, Descartes [Descartes 1629] commented on a universal grammar and dictionary that someone had been marketing commercially, with the promise that "anyone who learns this (universal) language, would also know all the others as dialects of it". Descartes 
found the idea naïve, and raised several arguments against it. However, he also gave his own suggestion of a universal language, such that it would

establish an order among all thoughts that can enter in the human spirit, in the same way as there is a natural order among numbers, and as one can learn in one day the names of all numbers up to infinity and write them in an unknown language, even though they are an infinity of different words...

The invention of this language depends on the true philosophy; for it is impossible otherwise to denumerate all thoughts of men and order them, or even distinguish them into clear and simple ones...

If anyone had well explained which are the simple ideas that are in the imagination of men, of which all that they think is composed ...then I would dare to hope for a universal language easy to learn, pronounce, and write and ... which would help judgement, representing all things to it so distinctly that error would be almost impossible. [Descartes 1629]

Descartes's suggestion is not widely known: the modern idea of universal language is usually traced back to Leibniz's characteristica universalis, a symbolic language permitting mechanized reasoning by means of a calculus ratiocinator. This proposal is from 1677, and advocates, like Descartes's, a mathematical notation such that the elements of the notation correspond to the elements of things and facts. The emergence of this idea in both Descartes and Leibniz is natural, given that they both made major contributions to mathematics with notational innovations as an important ingredient: analytic geometry in Descartes's case, and differential and integral calculus in Leibniz's.

The main aspect of a universal language for both of Descartes and Leibniz was that the notation would admit of a calculus to replace creative reasoning. A contemporary variant of the calculus idea is, of course, that a universal notation can be manipulated by a computer program, which can decide the correctness of judgements and - if the notation serves as bridge between languages - translate between languages. 


\subsection{Two Dimensions of Universality}

Speaking of a universal grammar, or of a universal language, we have to distinguish between two senses of universality:

Horizontal universality: generality across languages,

Vertical universality: generality across subject matters.

We chose "vertical" and "horizontal" mostly because we did not find better names, but they do suggest a major point we want to make, namely that these aspects are orthogonal. Therefore, to assess any proposal of and any argument against a "universal grammar", we have to find out which sense is meant.

What Roger Bacon wrote was explicitly about horizontal universality. Leibniz was explicit that he meant vertical universality. In Descartes's letter, both dimensions are mentioned.

The Sapir-Whorf hypothesis is a famous 20th century challenge of universality, and it is clearly about horizontal universality:

No two languages are ever sufficiently similar to be considered as representing the same social reality. The worlds in which different societies live are distinct worlds, not merely the same world with different labels attached. [Sapir 1929]

This point raised by Sapir is often discussed as a problem of translation between languages: not only is it difficult to find a translation from one language to another, but it may even happen that no translation exists, since the content expressed by the source language utterance has no counterpart in the target language.

Also vertical universality has important 20th century challenges. One consequence of Gödel's incompleteness proofs is that there cannot be a formal system that is complete for all mathematics - let alone for all subject matters, of which mathematics is but a fraction. And one of the important points in Wittgenstein's late philosophy is that there is no such thing as language, but just a collection of language games [Wittgenstein 1953]. Nor is there such a thing as the meaning of a word (simpliciter), but only its use in a language game. Now, an individual language game is a unit that has a set of rules that can possibly be formalized into a closed system; but the totality of language games cannot be formalized. 


\subsection{Cross-linguistic Language Games}

The main thesis of this paper is that

we can achieve horizontal universality but not vertical universality.

In other words, we can build cross-linguistic grammars on limited domains, or, "we can translate language games".

What we call a cross-linguistic language game corresponds to an area of multilingual activity and a tradition of translation, e.g. among scientists within one discipline, among employees within a multinational corporation, or among sportsmen practising the same sport. Of course, we have to leave it open whether horizontal universality covers all languages in the world: given a language game, we can only claim universality over those languages in which the game can be played.

Cross-linguistic equivalence based on language games is clearly different from the genetic relatedness of languages. There are several examples showing that shared cultural activities may be more important than genetic relatedness: Swedish (Germanic) and Finnish (Finno-Ugric) are largely intertranslatable - there is even a bilinguality legislation in Finland saying that all official documents must exist in both languages; on the other hand, the two Germanies after the Second World War were reported to be drifting apart linguistically. One criterion, or test, of horizontal equivalence is that:

it is possible to translate from one language to another.

In a sense, translatability between different languages fails very soon. Lots of famous examples can be found from Bible translation. For instance, there are so-called alphabetical Psalms, where subsequent verses begin with subsequent letters of the Hebrew alphabet. Translators have had different ambition levels in reproducing this feature in other languages. Normally, however, we speak of translation on some level of abstraction. A typical level is semantical: the translation of expressions as expressions for certain things. We thus arrive at a weaker criterion of horizontal equivalence:

it is possible to express the same things in the two languages.

\subsection{Defining a Level of Abstraction}

A natural way to define the semantic level of abstraction is to take as the starting point the things expressed — not the languages! In other words, 
one can start with a formalized, mathematical model of those things and see how it is reflected in languages - instead of starting with one of the languages and trying to find a model for that language.

This approach is opposite to the idea of formalization exercises that are customary in elementary logic teaching. Such an exercise consists in translating natural language sentences into logical formulae, with a painful awareness that something is getting lost. For instance, the meaning of love is felt to be lost when formalizing John loves Mary as love(John, Mary). It is much less painful to start from the formula and ask how to express it in natural language! The logic textbook of Kalish and Montague [Kalish \& Montague 1964] is one of the few that contains exercises in this direction: in retrospect, it can well be seen as a precursor of Montague grammars (see next section).

\section{Multilingual Grammars}

\subsection{Curry's Model of Multilingual Grammar}

Curry published in [Curry 1963] a linguistic paper in which he distinguished between the tectogrammatical structure and the phenogrammatical structure of a languge. The tectogrammatical structure has to do with how expressions are divided into meaningful parts; the phenogrammatical structure has to do with what expressions look like. It is the tectogrammatical structure to which, for instance, semantics applies; that subsequent verses of a Psalm begin with subsequent letters is rather a phenogrammatical fact.

Together with the distinction, Curry formulated a program for multilingual grammars: such a grammar would have one tectogrammatical structure and many phenogrammatical structures. For Curry, the tectogrammatical structure was to be described by combinatory logic. This gives the following picture:

tectogrammatical phenogrammatical

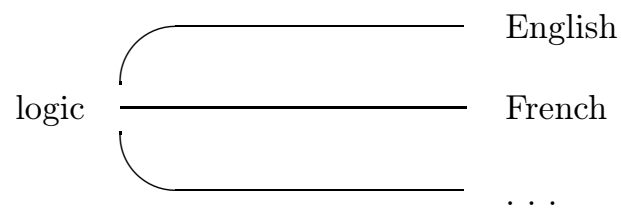


The semantic aspect of Curry's model was developed in great detail by Montague ([Montague 1974]; the background of Montague's work in Curry's was pointed out by Dowty [Dowty 1982]). Neither Curry nor Montague pursued the multilingual aspect, but it is present in the works of Shaumyan [Shaumyan 1965] and Desclés [Desclés 1994], both building on Curry, and in a later translation project based on Montague grammar, Rosetta [Rosetta 1995].

It is not clear if Curry and Montague also supported vertical universality; Montague, at least, seems to have thought that higher-order predicate calculus with modal operators was sufficient for everything; more accurately, that the semantics of English consists of giving truth conditions to all English sentences in a model of this calculus.

\subsection{Grammatical Framework}

The formalism GF (Grammatical Framework, [Ranta 2004]) adopts from Curry and Montague the use of logic as a language-independent representation level. The logic that GF uses is constructive type theory [MartinLöf 1984], in the so-called higher-level format first published in [Nordström et al. 1990]. For Curry's tectogrammatical and phenogrammatical structure, GF uses the computer science terminology of abstract syntax and concrete syntax (Landin 1966). The modified picture of Curry's model is the following:

abstract syntax concrete syntax

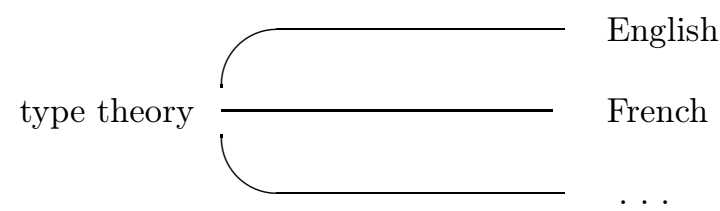

As is standard in the study of programming languages, semantic operations such as type checking and evaluation are performed on the abstract syntax level. Concrete syntax is the syntax that is printed and parsed, and that the language users see. For instance, a variable declaration can have different notations, such as those of Pascal and C:

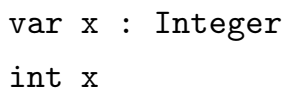

The abstract syntax is however the same in the two cases: what matters is that a variable $\mathrm{x}$ of integer type is declared. 


\subsection{The Framework Idea}

Unlike Curry's combinatory logic and Montague's intensional logic, the type theory of GF is not one single calculus, but a framework. This idea comes from Logical Frameworks [Harper et al. 1986], which are type theories used as metatheories, for describing and implementing individual logical calculi and mathematical theories.

When mathematics is formalized in a logical framework, there is no need to have one unifying theory of mathematics in terms of which everything is defined (such as set theory): the framework is a weak calculus, and its power comes from the individual theories and calculi that are defined within it. The gain of using a logical framework to define logics, rather than defining them directly, is that the framework can take care of operations of "book-keeping" character, such as inference rule application, substitution, and variable binding. The gain achieved when implementing logic on computers can be considerable.

\subsection{GF Judgements and Grammars}

As for abstract syntax, GF is just another logical framework. The abstract syntax part of a grammar consists of judgements of the following two forms:

$$
\begin{aligned}
& \text { cat } C \\
& \text { fun } f: A-C \text { is a category } \\
& \text { fun a function of type } A
\end{aligned}
$$

(Yet another form is definitions of functions, which we will not use.)

What is not present in logical frameworks, are judgements for defining concrete syntax. The most important forms are the following, needed for each category $C$ and function $f$ defined in the abstract syntax:

$$
\begin{aligned}
& \text { lincat } C=T-C \text { has the linearization type } T \\
& \text { lin } f=t
\end{aligned}
$$

A grammar is a pair

$$
<\mathcal{A}, \mathcal{C}>
$$

of abstract and concrete syntax. A multilingual grammar is a pair

$$
<\mathcal{A},\left\{\mathcal{C}_{1}, \ldots, \mathcal{C}_{n}\right\}>
$$

of an abstract syntax and a set of concrete syntaxes. 


\subsection{Type Checking, Linearization, and Parsing}

A GF grammar is a purely declarative definition of a language. But the formalism is so defined that it is always possible to derive the following algorithms automatically:

Type checking decides whether a given tree in abstract syntax has a given type.

Linearization takes a tree in abstract syntax to an object in the corresponding linearization type.

Parsing takes a string into a set of abstract syntax trees (empty set: parsing fails; more than one element: the string is ambiguous).

The type checking algorithm is inherited from logical frameworks. The linearization algorithm can be straightforwardly derived from the linearization rules, and it is similar to expression evaluation in $\lambda$-calculus and functional programming. The parsing algorithm is based on a nontrivial inversion of the linearization rules. Altogether, deriving these algorithms from a declarative source gives an implementation gain analogous to implementing logics in a logical framework.

As a corollary of linearization and parsing, a multilingual grammar automatically has

Translation from $\mathcal{C}_{i}$ to $\mathcal{C}_{j}$ is parsing from $\mathcal{C}_{i}$ followed by linearization to $\mathcal{C}_{j}$.

The translation goes via $\mathcal{A}$, which guarantees semantic equivalence but does not preclude ambiguity.

\subsection{Example: A Fragment of Arithmetic}

GF has been applied to many different domains of language, ranging from software specifications [Burke \& Johannisson 2005] to spoken dialogue systems [Ranta \& Cooper 2004]. We will here illustrate GF with a very small multilingual grammar, whose subject matter is arithmetic. The abstract syntax introduces the categories of natural numbers and propositions, the number zero, and the predicate that a number is even.

cat Nat

cat Prop 
fun Zero : Nat
fun Even : Nat $\rightarrow$ Prop

An English concrete syntax can be given as follows:

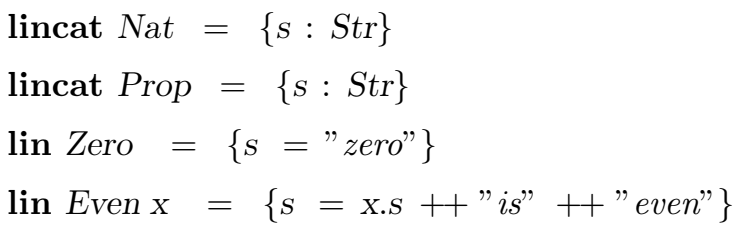

The linearizations are records which may have many different fields. In the simplest case, as here, there is just one field holding a string. As we will see, much of the difference between concrete syntaxes comes from what types of records are assigned to each category.

Using the abstract syntax above, we can form the tree

\section{Even Zero}

of type Prop. Using the concrete syntax, we compute the linearization of this proposition:

$$
\{s=" \text { zero" }++" \text { is" ++ "even" }\}
$$

\subsection{Arithmetic in French}

Abstract syntax abstracts away from features belonging to concrete syntax. One of these features is morphological variation, which is very different in different languages. To describe morphological variation in the concrete syntax, we introduce a form of judgement permitting the definition of parameter types. For instance, even in a tiny fragment of arithmetic, the French concrete syntax will need the parameters of mood and gender:

$$
\begin{aligned}
& \text { param Mod }=\text { Ind } \mid \text { Subj } \\
& \text { param Gen }=\text { Masc } \mid \text { Fem }
\end{aligned}
$$

Expressions for natural numbers are, linguistically, noun phrases, and have an inherent gender, that is, a gender element belonging to the record as an extra field. 


$$
\begin{aligned}
& \text { lincat } N a t=\left\{\begin{array}{lll}
s & : & \text { Str } \\
g & : & \text { Gen }
\end{array}\right\} \\
& \operatorname{lin} \text { Zero }=\left\{\begin{array}{l}
s=\text { "zéro" } \\
g=\text { Masc }
\end{array}\right\}
\end{aligned}
$$

An inherent gender in GF is a formalization of gender information such as given in a traditional dictionary.

Expressions for propositions are, linguistically, sentences. Their formation depends on a mood parameter, which is passed to the verb of the sentence. In a predication sentence formed by using an adjective, the adjective is inflected in the gender of the subject noun phrase.

$$
\begin{aligned}
& \text { lincat Prop }=\{s: \operatorname{Mod} \Rightarrow \text { Str }\} \\
& \text { lin Even } x= \\
& \text { let pair }=\text { table }\left\{\begin{array}{l}
\text { Masc } \Rightarrow \text { "pair" } \\
\text { Fem } \Rightarrow \text { "paire" }
\end{array}\right\} \text { x.g in } \\
& \left\{s=\text { table }\left\{\begin{array}{ll}
\text { Ind } \Rightarrow \text { x.s }+ \text { "est" + pair } \\
\text { Subj } \Rightarrow \text { x.s }++ \text { soit" + + pair }
\end{array}\right\}\right\}
\end{aligned}
$$

The linearization of a sentence is thus a table that gives a value to each of the parameters of a parameter type. This notion is a formalization of inflection tables occurring in traditional grammar.

\subsection{Translating between English and French}

The following examples (from an enlarged fragment) show how French uses the gender to generate different forms of the adjective.

zero is even $=$ zéro est pair

the sum of zero and zero is even $=$ la somme de zéro et de zéro est paire

The following example shows a French construction requiring subjunctive mood of a subordinate clause.

there exists an $x$ such that $x$ is even $=i l$ existe un $x$ tel que $x$ soit pair

All the translations above are obtained via parsing into abstract syntax and subsequent linearization into concrete syntax. They work, of course, in both directions. 


\section{The Unity of a Language}

\subsection{Unity of Languages Lost?}

If we write the grammar of each language game separately, we will end up having no common structure in the sentences zero is even, smallpox is contagious, and the weather is beautiful, unless we find a language game that covers them all. This is counterintuitive: one would prefer to say that all of these expressions have the same syntactic structure, which is that of a sentence formed by adjectival predication.

A more general aspect of the problem is: if we can only describe language games, then there is no such thing as English or French language. This is counterintuitive: when I learn French, there is something unified that I learn - not just a set of distinct language games, but something that enables me to play any of my old language games in a new language. What is this thing?

\subsection{Resource Grammars}

The way we can do justice to the unity of languages, in the ordinary sense, is by raising the level of abstraction in concrete syntax. Instead of defining linearization directly into strings and records of strings, we use intermediate syntactic structures. For instance, instead of defining

$$
\operatorname{lin} \operatorname{Even} x=\{s=x . s++" i s "++" \text { even" }\}
$$

we use an adjectival predication function and a lexical item representing the adjective even:

$$
\text { lin Even } \mathrm{x}=\text { PredAP } \mathrm{x} \text { even }
$$

These intermediate structures are collected into a resource grammar, whose aim is to define a natural language, such as English, instead of a language game. A resource grammar is built by considering the language independently of the various language games in which it might be used. Although it is defined in GF just like the semantically motivated structures, no semantic explanation is expected. On the other hand, the linearization rules of a resource grammar will be intuitive and immediate, since the grammar has been formulated by directly observing the concrete usage of the language. 


\subsection{Example Resource Grammar}

The abstract syntax of a resource grammar is built from traditional linguistic categories and rules. In the GF resource grammar project [Ranta 2005], we have found it useful to have, among other things, sentences, noun phrases, and adjectival phrases.

cat $S ; N P ; A P$

The functions formalize rules that combine such phrases, e.g.

fun PredAP : $N P \rightarrow A P \rightarrow S$

tells that a noun phrase and an adjectival phrase can be combined into a sentence.

To give an English concrete syntax to this resource grammar, we first define the agreement features of noun phrases and verb phrases, constructed from number and person:

$$
\begin{aligned}
& \text { param Num }=S g \mid P l \\
& \text { param Person }=P 1|P 2| P 3 \\
& \text { param } A g r=A g N u m \text { Person }
\end{aligned}
$$

Then we define the linearization types of syntactic categories and the linearization rules of the syntactic functions:

$$
\begin{aligned}
& \text { lincat } S=\{s: S t r\} \\
& \text { lincat } N P=\left\{\begin{array}{lll}
s & : & \text { Str } \\
a & : & A g r
\end{array}\right\} \\
& \text { lincat } A P=\{s: S t r\} \\
& \text { lin PredAP np adj = } \\
& \{s=n p . s++ \\
& \text { table } \left.\left\{\begin{array}{lll}
\operatorname{Ag} \operatorname{Sg} P 1 & \Rightarrow & \text { "am" } \\
\operatorname{Ag} \operatorname{Sg} P 3 & \Rightarrow & \text { is" } \\
- & \Rightarrow & \text { are" }
\end{array}\right\} \text { !np.a }+ \text { adj.s }\right\}
\end{aligned}
$$

Finally, the resource grammar may also have a lexicon, which defines the linguistic properties of words without paying notice to their semantics. For instance, the adjective even is unlikely to have a uniform semantic interpretation, but it does have a uniform shape as an adjective.

$$
\begin{aligned}
& \text { fun even : } A P ; \operatorname{lin} \text { even }=\{s=" \text { even" }\} \\
& \text { fun zero : } N P ; \operatorname{lin} \text { zero }=\left\{\begin{array}{l}
s=" \text { zero" } \\
a=A g S g P 3
\end{array}\right\}
\end{aligned}
$$




\subsection{Using Resource Grammars}

In contrast to resource grammars, we use the term application grammar for a grammar describing a semantically interesting language game. To define the concrete syntax of an application grammar, we can now give its linearization types and rules in terms of the categories and functions of the resource. In English arithmetic, for instance, we define:

$$
\begin{aligned}
& \text { lincat Prop }=S ; \text { lincat } N a t=N P \\
& \text { lin Zero }=\text { zero } ; \text { lin Even } \mathrm{x}=\text { PredAP } \mathrm{x} \text { even }
\end{aligned}
$$

Structures and words in the resource grammar do not have any deeper semantic meaning. In a sense, their semantics is given indirectly, through the different uses to which they are put in different application grammars - in other words, through their uses in different language games. For instance, the adjective even is used to linearize the concept of divisibility by 2 in some applications, and the concept of smoothness of a surface in others.

\subsection{Shared Structures of Resource Grammar}

Even though concrete syntax differs a lot from one natural language to another, there is surprisingly much of the abstract syntax of resource grammars that can be shared. Many languages have categories like sentence, noun phrase, and adjectival phrase, and rules like adjectival predication, if we ignore differences of parametric variation and word order. The GF resource grammar library (Ranta 2005) thus implements a comprehensive set of grammatical categories and rules for ten languages.

For instance, the above fragment can be easily reused for French, by just redefining the linearization types and rules. The (abstract) linearization types assigned to semantic types are the same as in English,

$$
\text { lincat Prop }=S \text {; lincat } N a t=N P
$$

The French linearization rules are

$$
\text { lin Zero }=\text { zéro } ; \text { lin Even } \mathrm{x}=\text { PredAP } \mathrm{x} \text { pair }
$$

If we have a German implementation of the resource, we can write

$$
\text { lin Zero }=\text { null } ; \text { lin Even } \mathrm{x}=\text { PredAP } \mathrm{x} \text { gerade }
$$


The lexical items zero, even, zéro, pair, null, and gerade are defined individually in the resource lexica of each language. But the syntactic structure PredAP is defined for every language. On this abstraction level, we can thus conclude with the anonymous 12th century author that the difference between languages "arises from the difference of words and their formations".

\subsection{Differences in Concrete Syntax Structure}

We have seen that raising the abstraction level in concrete syntax makes it possible for different languages to share rules even there, and not only in the semantic-level abstract syntax. But of course, we should not expect this always to hold. A well-known example from translation is the verb miss, where translating between English and French requires swapping the subject and the object:

$x$ misses $y=y$ manque $\grave{a} x$

Only the abstract syntax predicate Miss can be shared: concrete syntax uses different structures. The English and French linearization rules are

$$
\begin{aligned}
& \operatorname{lin} \text { Miss } \mathrm{x} y=\operatorname{PredVP} \mathrm{x}(\mathrm{ComplV} 2 \text { miss } \mathrm{y}) \\
& \operatorname{lin} \text { Miss } \mathrm{x} y=\operatorname{PredVP} \mathrm{y}(\mathrm{ComplV} 2 \text { manquer } \mathrm{x})
\end{aligned}
$$

In this example, it is no longer possible to say that just the words are different in the two languages.

\section{Conclusion}

We have not tried to define a universal grammar that is both vertical and horizontal, i.e. covers everything that can be said in any language. However, we have found it possible to achieve horizontal universality in semantically limited domains, by using type-theoretical abstract syntax. Moreover, we have found a meager variant of vertical universality, resource grammars, which cover syntax but no semantics. 


\title{
References
}

\author{
Burke, D. A. \& Johannisson, K
}

2005 Translating Formal Software Specifications to Natural Language / A Grammar-Based Approach, in Blache, P., Stabler, E., Busquets, J. \& Moot, R. (eds), Logical Aspects of Computational Linguistics (LACL 2005). LNCS/LNAI 3402, Heidelberg: Springer, $51-66$.

Curry, H. B.

1963 Some Logical Aspects of Grammatical Structure, in Jakobson, R. (ed), Structure of Language and its Mathematical Aspects: Proceedings of the Twelfth Symposium in Applied Mathematics, American Mathematical Society, 56-68.

Descartes, R.

1629 Letter to Mersenne, 20 November 1629. Published in French translation in Descartes, Euvres et lettres, by A. Bridoux (ed.), Paris: Gallimard, 911-915.

Desclés, J. P.

1994 Langages applicatifs, langues naturelles et cognition. Paris: Hermès.

DowTY, D.

1982 Grammatical Relations and Montague Grammar, in P. Jakobson \& G. K. Pullum (eds.), The Nature of Syntactic Representation, Dordrecht: D. Reidel, 79-130.

GiLson, É.

1922 La philosophie au moyen âge, Paris: Payot.

Harper, R., Honsell, F., \& Plotkin, G.

1993 A Framework for Defining Logics, Journal of the ACM, 40 (1), 143-184.

Kalish, D. \& Montague, R.

1964 Logic: Techniques of Formal Reasoning, New York: Harcourt Brace.

LANDIN, P.

1966 The Next 700 Programming Languages, Communications of the ACM, 9, 157-166. 
LYONS, J.

1968 Introduction to Theoretical Linguistics, Cambridge: Cambridge University Press.

MARTIN-LÖF, P.

1984 Intuitionistic Type Theory, Napoli: Bibliopolis.

Montague, R.

1974 Formal Philosophy, New Haven: Yale University Press, Collected papers edited by R. Thomason.

Nordström, B., Petersson, K., \& Smith, J. M.

1990 Programming in Martin-Löf's Type Theory. An Introduction, Oxford: Oxford University Press.

RANTA, A.

2004 Grammatical Framework: A Type-Theoretical Grammar Formalism, The Journal of Functional Programming, 14 (2), 145-189.

Ranta, A. \& Cooper, R.

2004 Dialogue Systems as Proof Editors, Journal of Logic, Language and Information, 13, 225-240.

RANTA, A.

2005 Modular Grammar Engineering in GF, Research on Language and Computation, to appear.

Rosetta, M. T.

1994 Compositional Translation, Dordrecht: Kluwer.

SAPIR, E.

1929 The Status of Linguistics as a Science, in E. Sapir (1958), Culture, Language and Personality, D. G. Mandelbaum (ed.), Berkeley, CA: University of California Press.

SHAUMYAN, S. K.

1965 Strukturnaya linguistika, Moscow: Nauka.

Wittgenstein, L.

1953 Philosophical Investigations, Oxford: Basil Blackwell. 